\title{
Acknowledgement to Reviewers of Universe in 2019
}

\author{
Universe Editorial Office
}

MDPI, St. Alban-Anlage 66, 4052 Basel, Switzerland

Published: 20 January 2020

The editorial team greatly appreciates the reviewers who have dedicated their considerable time and expertise to the journal's rigorous editorial process over the past 12 months, regardless of whether the papers are finally published or not. In 2019, a total of 225 papers were published in the journal, with a median time to first decision of 22 days and a median time from submission to publication of 44 days. The editors would like to express their sincere gratitude to the following reviewers for their generous contribution in 2019:

Aarts, Gert

Abraham, Zulema

Adams, Fred

Adams, Jenni

Agullo, Ivan

Aichelin, Jörg

Akiyama, Kazunori

Alodjants, Alexander

Alves Batista, Rafael

Andrew, Keith

Arbuzov, Andrei

Aros, Rodrigo

Arzano, Michele

Asada, Hideki

Attems, Maximilian

Ayriyan, Alexander

Babichev, Eugeny

Bahamonde, Sebastián

Balakin, Alexander B.

Bamba, Kazuharu

Bambi, Cosimo

Barberio, Elisabetta

Barnacka, Anna

Barnich, Glenn

Barrau, Aurelien

Barrow, John
Bartelmann, Matthias

Bass, Steven D.

Beau, Mathieu

Beccaria, Matteo

Beck, Douglas H.

Belli, Pierluigi

Bellwied, Rene

Berezhiani, Zurab

Bernuzzi, Sebastiano

Bhattacharya, Tanmoy

Bicknell, Geoffrey

Bicudo, Pedro

Bielčík, Jaroslav

Biernat, Elmar

Biesiada, Marek

Bietenholz, Wolfgang Peter

Bisnovatyi-Kogan, Gennady S.

Björn, Schenke

Blaschke, David

Blasone, Massimo

Blázquez-Salcedo, Jose Luis

Blok, Boris

Bodendorfer, Norbert

Bojowald, Martin

Bondarescu, Ruxandra

Bonga, Beatrice 
Bopp, Fritz W.

Bower, Richard

Bozapalidis, Symeon

Bradley, Michael

Brahma, Suddhasattwa

Brandenberger, Robert H.

Bravina, Larissa

Bronnikov, Kirill

Brown, Ethan

Bulnes, Francisco

Burdin, Sergey

Cacciatori, Sergio

Cai, Yi-Fu

Cao, Zhoujian

Capolupo, Antonio

Carrozza, Sylvain

Casadio, Roberto

Catena, Riccardo

Celi, Alessio

Cembranos, Jose

Chamoun, N.

Chang, Jin

Chen, Chuan-Hung

Chepel, Vitaly

Choi, Suyong

Choudhury, Sayantan

Christakoglou, Panagiotis

Cleaver, Gerald

Cohen, Elihau

Cohen, Thomas

Combrinck, Ludwig

Contopoulos, Ioannis

Corda, Christian

Cortes, Jose

Craig, Nathaniel

Crkovska, Jana

Csanád, Máté

Csörgő, Tamás

Dabrowski, Mariusz

D'Agostino, Rocco

Dai, Liang

Danehkar, Ashkbiz
Das, Saurya

Das, Upasana

Davies, Paul C. W.

De Diego, José A.

De Freitas-Pacheco, José Antonio

De Martino, Ivan

De Paolis, Francesco

Degollado, Juan Carlos

Del Popolo, Antonino

Del Zanna, Luca

Deng, Xue-Mei

Deppman, Airton

Dexheimer, Veronica

Di Marco, Alessandro

Dialektopoulos, Konstantinos

Doi, Takahiro

Dremin, Igor M.

Dulat, Falko

Dvali, Georgi

Dymnikova, Irina

Efroimsky, Michael

Eichhorn, Astrid

Elfner, Hannah

Escobedo Espinosa, Miguel Ángel

Essén, Hanno

Etzion, Erez

Evans, Justin

Fabris, Júlio

Fan, Xilong

Fendt, Christian

Feoli, Antonio

Fernando, Sharmanthie

Ferreira, Ricardo José Zambujal

Fischbach, Ephraim

Florkowski, Wojciech

Fortin, Morgane

Foschini, Luigi

Frey, Sandor

Friedman, Yaakov

Gaitanos, Theodoros

García, J Antonio

Garzelli, Maria Vittoria 
Gasperini, Maurizio

Gazeau, Jean-Pierre

Gergely, László

Gerosa, Davide

Ghiglieri, Jacopo

Gies, Holger

Ginsburg, Idan

Giunti, C.

Glaser, Lisa

Gong, Yungui

González-Díaz, Diego

Gorbenko, Victor

Gorda, Tyler

Gourgouliatos, Konstantinos

Gralla, Samuel E.

Grunfeld, Ana Gabriela

Gubitosi, Giulia

Guendelman, Eduardo

Gupt, Brajesh

Haggard, Hal

Halliwell, Jonathan

Hanauske, Matthias

Hatfield, Peter

Hawley, John

Hehl, Friedrich W.

Helayel-Neto, J. A.

Heller, Michał

Hendi, Seyed Hossein

Herdeiro, Carlos

Hidalgo, Gabriel Flores

Higinbotham, Douglas

Hiramatsu, Takashi

Hobill, David

Hoff Da Silva, Julio M.

Hofmann, Ralf

Hohmann, Manuel

Horwitz, Lawrence

Huang, Wister

Huber, Markus Q.

Ivanov, Yu. B.

Jegerlehner, Fred

Johnson-McDaniel, Nathan
Jones, Benjamin

Jordan, Andrew

Joyce, Michael

Jurčišinová, Eva

Jurić, Tajron

Kaimakamis, George

Kajantie, Keijo

Kamenshchik, Alexander Yu.

Kaminker, Alexander D.

Karpenko, Iurii

Karsch, Frithjof

Kashi, Amit

Keresztes, Zoltan

Khaw, Kim Siang

Khurshudyan, Martiros

Kibler, Maurice R.

Kim, Yong-Su

Kimura, Rampei

Kirillov, Alexander

Kleidis, Kostas

Kobayashi, Tsutomu

Kolomeitsev, Evgeni

Kopeikin, Sergei

Kosloff, Ronnie

Kotanjyan, Anna

Kotikov, Anatoly

Kovács, Péter

Kozlov, Mikhail

Krajewski, Thomas

Krämer, Manuel

Krishnan, Chethan

Krizek, Filip

Krssak, Martin

Kuang, Xiao-Mei

Lagos, Macarena

Lambiase, Gaetano

Lavagno, Andrea

Lebiedowicz, Piotr

Lecian, Orchidea Maria

Lee, Chung-Chi

Lee, Hayden

Lepe, Samuel 
Létang, Jean Michel

Li, Liang

Li, Shirley Weishi

Liberali, Valentino

Liberati, Stefano

Licata, Ignazio

Lichtenegger, Herbert

Lin, Lap Ming

Lingam, Manasvi

Liss, Tony $\mathrm{M}$.

Liu, Fu-Hu

Liu, Xiang

Livine, Etera

Logoteta, Domenico

LoSecco, John M.

Louko, Jorma

Ludu, Andrei

Lunin, Oleg

Luongo, Orlando

Luzum, Matthew

Magdy, Niseem

Maia, M. D.

Maluf, José Wadih

Marciano, Antonino

Mariani, Camillo

Marochnik, Leonid

Martin-Benito, Mercedes

Martín-Moruno, Prado

Mashhoon, Bahram

Matthew, Durham J.

Mavromatos, Nick E

Mena-Marugan, Guillermo A.

Menezes, Gabriel

Miatto, Filippo M.

Mielczarek, Jakub

Mielke, Eckehard

Mifsud, Jurgen

Millington, Peter

Miramonti, Lino

Misra, Ranjeev

Mitsou, Vasiliki

Mizuno, Yosuke
Modanese, Giovanni

Moffat, John W.

Mondal, Tushar

Monthus, Cecile

Moosa, Mudassir

Mortemousque, Pierre-Andre

Moustakidis, Charalampos

Mück, Wolfgang

Nagy, Márton

Nakayama, Yu

Nampalliwar, Sourabh

Nara, Yasushi

Nättilä, Joonas

Neiman, Yasha

Nester, James M.

Nicolas, Chamel

Noiri, Akito

Nomura, Yasunori

Novák, Tamás

Nowakowski, Marek

Odintsov, Sergei

Oikonomou, Vasileios

Oles, Andrzej M.

Onishchenko, Andrei

Osato, Ken

Overduin, James

Ovgun, Ali

Pagani, Carlo

Pagliara, Giuseppe

Paic, Guy

Pajares, Carlos

Pal, Bilas K.

Papp, Gábor

Paraoanu, Gheorghe Sorin

Park, Inyong

Paschos, Emmanuel A.

Pasechnik, Roman

Passante, Roberto

Pásztor, Gabriella

Pelster, Axel

Percacci, Roberto

Pereira, Antonio 
Perlick, Volker

Petrov, Albert

Piattella, Oliver

Picanço, Rodrigo

Piedipalumbo, Ester

Pinčák, Richard

Poletti, Dario

Povstenko, Yuriy

Pranzetti, Daniele

Pratt, Scott

Procureur, Sébastien

Providencia, Constança

Pruneau, Claude

Pullin, Jorge

Qiu, Jian-Wei

Queiroz, Farinaldo

Radi, Amr

Radice, David

Rahvar, Sohrab

Raidal, Martti

Ramirez, Cupatitzio

Rampf, Cornelius

Ratti, Claudia

Reece, Matt

Rees, Martin

Reinhardt, Hugo

Rey, Soo-Jong

Ringer, Felix

Robbins, Daniel

Robles-Perez, Salvador

Rocha, Roldão Da

Rogatko, Marek

Rossi, Giancarlo

Rubiera-Garcia, Diego

Rubin, David

Rueda, Jorge

Rybczyński, Maciej

Ryblewski, Radoslaw

Ryskin, Mikhail G.

Saal, Margus

Sahlén, Martin

Saikawa, Kenichi
Sanchez, Miguel

Santoni, Luca

Savelova, Elena

Sbitnev, Valery

Scampoli, Paola

Schaefer, Bernd-Jochen

Schäfer, Björn Malte

Schee, Jan

Schmitt, Andreas

Schulze-Makuch, Dirk

Sedrakian, Armen

Senjanovic, Goran

Shalyt-Margolin, Alexander

Shao, Lijing

Shapiro, Ilya

Shen, Chun

Sheng, Yu-Bo

Shore, Graham

Shtanov, Yuri

Siklér, Ferenc

Singleton, Douglas

Sitta, Mario

Słodkowski, Marcin

Smirnov, Alexei

Soda, Jiro

Solà, Joan

Speziale, Simone

Steinhaus, Sebastian

Stergiou, Andreas

Stuchlík, Zdeněk

Sultana, Joseph

Sun, Jia Rui

Sushkov, Sergei

Tawfik, Abdel Nasser

Tempo, David

Tenkanen, Tommi

Tessarotto, Massimo

Tetradis, Nikolaos

Thompson, Lee

Toriumi, Reiko

Torrieri, Giorgio

Tsukamoto, Naoki 


\begin{tabular}{ll} 
Tsupko, Oleg & Wimberger, Sandro \\
Typel, Stefan & Wlodarczyk, Zbigniew \\
Ulhoa, Sérgio Costa & Wolf, Marek \\
Vacca, Gian Paolo & Wu, Dufan \\
Vagnozzi, Sunny & Xiaofeng, Luo \\
Valcarcel, Jorge Gigante & Xu, Donglian \\
Van Den Hoogen, Robert & Yang, Run-Qiu \\
Van Hees, Hendrik & Yasusada, Nambu \\
Velten, Hermano & Yokomizo, Nelson \\
Venanzoni, Graziano & Yu, Xiaoquan \\
Vento, Vicente & Yuan, Feng \\
Vergara, José David & Zabrodin, Evgeny \\
Vertesi, Robert & Zaccolo, Valentina \\
Vitev, Ivan & Zakharov, Alexander F. \\
Vittino, Andrea & Zanelli, Jorge \\
Vogt, Ramona & Zaninetti, Lorenzo \\
Voskresensky, Dmitry N. & Zárate, Laura Rosales \\
Vovchenko, Volodymyr & Zavala, Jesus \\
Wahlberg, Hernan & Zdunik, Julian Leszek \\
Wang, Michael H.L.S. & Zhidenko, Olexandr \\
Wang, Sai & Zilhao, Miguel \\
Wei, Hanyu & Zimdahl, Winfried \\
Werner, Klaus & Zinoviev, Yurii \\
Wieland, Wolfgang & Zipfel, Antonia \\
Wiggins, Brandom & Zlosnik, Thomas \\
Will, Clifford M. & Zou, Yuanchuan \\
\hline &
\end{tabular}

(C) 2020 by the authors. Licensee MDPI, Basel, Switzerland. This article is an open access article distributed under the terms and conditions of the Creative Commons Attribution (CC BY) license (http://creativecommons.org/licenses/by/4.0/). 